\title{
KÄHLER MANIFOLDS WITH CURVATURE BOUNDED FROM ABOVE BY A DECREASING FUNCTION
}

\author{
MITSUHIRO ITOH
}

\begin{abstract}
Let $M$ be a simply connected complete Kähler manifold. If $M$ has curvature bounded from above by a certain positive decreasing function, then it is a Stein manifold, diffeomorphic to a euclidean space. This fact is a generalization of the well-known propositions for complete manifolds of nonpositive curvature and is shown by the aid of a Rauch comparison theorem for conjugate points together with a comparison theorem of Siu and Yau with respect to the Hessian of distance functions.
\end{abstract}

1. The aim of this paper is to show the following.

THEOREM. Let $(M, g)$ be a simply connected complete Riemannian manifold. If there is a point $o$ in $M$ such that along each geodesic $c$ emanating from $o$ parametrized by arc-length the sectional curvature $K_{\sigma(t)}$ of any plane $\sigma(t)$ at $c(t)$ $(t \geq 0)$ satisfies

$$
K_{\sigma(t)} \leqslant F(t),
$$

then $M$ is diffeomorphic to a euclidean space. Moreover if $(M, g)$ is Kählerian, then $M$ is a Stein manifold.

The function $F(t)$ is defined by

$$
F(t)=\operatorname{Min}\left\{\frac{1}{\cosh ^{2} t}, \frac{\sinh ^{2} t-\log \left(\cosh ^{2} t\right)}{2 \cosh ^{2} t\left\{\log \left(\cosh ^{2} t\right)\right\}^{2}}\right\} .
$$

It is positive and piecewise smooth.

The Theorem is a generalization of the well-known propositions; a simply connected complete Riemannian manifold with nonpositive curvature is diffeomorphic to a euclidean space and if it is Kählerian then it is a Stein manifold [1], [5].

Geometrical meaning of $F$ is as follows. It is known that a complex $n$-vector space $\mathbf{C}^{n}$ admits a complete Kähler metric

$$
\tilde{g}=\sum \frac{\partial f\left(|z|^{2}\right)}{\partial z^{i} \partial \bar{z}^{j}} d z^{i} d \bar{z}^{j}, \quad f(x)=\int_{0}^{x} \frac{1}{s} \log (1+s) d s,
$$

which has positive curvature [2]. We denote by $F(t)$ the infimum of the sectional curvatures at a point with distance $t$ from the origin 0 in $\mathbf{C}^{n}$ with

Received by the editors June 23, 1978.

AMS (MOS) subject classifications (1970). Primary 53C55; Secondary 32E10.

Key words and phrases. Sectional curvature, comparison theorem, Stein manifold. 
respect to $\tilde{g}$. Then (*) is equivalent to

$$
K_{\sigma(t)} \leqslant K_{\tilde{\sigma}(t)}
$$

for the sectional curvatures $K_{\sigma(t)}$ and $K_{\tilde{\sigma}(t)}$ of planes $\sigma(t)$ and $\tilde{\sigma}(t)$ at $c(t)$ and $\tilde{c}(t)$ respectively, where $\tilde{c}(t)$ is an arbitrary geodesic emanating from $\tilde{o}$ parametrized by arc-length in $\mathbf{C}^{n}$.

ReMARK. The function $F$ is rapidly decreasing since $F(t) \sim e^{-2 t}$ at infinity. The following is proposed; does there exist $F$ of order $O\left(t^{\alpha}\right)(\alpha<0)$ at infinity such that the Theorem holds with respect to this $F$ ?

2. We will describe explicitly geodesics emanating from $\tilde{o}$, the distance function $\tilde{r}$ and the infimum of the sectional curvatures of $\left(\mathbf{C}^{n}, \tilde{g}\right)$. At first we will derive these in the case where $\tilde{g}$ is given by a general smooth potential function $f$, that is, $g=\sum \partial f\left(|z|^{2}\right) / \partial z^{i} \partial \bar{z}^{j} d z^{i} d \bar{z}^{j}$. Note that by the definition of $\tilde{g}$ each spherical symmetric transformation is an isometry. Since

$$
\tilde{g}_{i j}(z)=f^{\prime}\left(|z|^{2}\right) \delta_{i j}+f^{\prime \prime}\left(|z|^{2}\right) \bar{z}^{i} z^{j}, \quad 1<i, j<n,
$$

the coefficients $\tilde{\Gamma}_{j k}^{i}(z)$ of the Riemannian connection is written as

$$
\begin{aligned}
\tilde{\Gamma}_{j k}^{i}(z)= & \frac{f^{\prime \prime}\left(|z|^{2}\right)}{f^{\prime}\left(|z|^{2}\right)}\left(\bar{z}^{k} \delta_{i j}+\bar{z}^{j} \delta_{i k}\right) \\
& +\frac{f^{\prime}\left(|z|^{2}\right) f^{\prime \prime \prime}\left(|z|^{2}\right)-2\left\{f^{\prime \prime}\left(|z|^{2}\right)\right\}^{2}}{f^{\prime}\left(|z|^{2}\right)\left\{f^{\prime}\left(|z|^{2}\right)+|z|^{2} f^{\prime \prime}\left(|z|^{2}\right)\right\}} z^{i} \bar{z}^{j} \bar{z}^{k}, \quad 1<i, j, k<n .
\end{aligned}
$$

Since

$$
\tilde{\Gamma}_{11}^{1}(z)=\frac{2 f^{\prime \prime}\left(|z|^{2}\right)+|z|^{2} f^{\prime \prime \prime}\left(|z|^{2}\right)}{f^{\prime}\left(|z|^{2}\right)+|z|^{2} f^{\prime \prime}\left(|z|^{2}\right)} \bar{z}^{1}
$$

and $\tilde{\Gamma}_{11}^{i}(z)=0$ for $i>1$ on the linear subspace $L=\left\{z=\left(z^{i}\right) ; z^{i}=0, i>1\right\}$ of $\mathbf{C}^{n}$, a curve $c(t)=(x(t), 0, \ldots, 0)$ lying on $L$ and starting at $\tilde{o}$ is a geodesic if $x(t)$ satisfies

$$
\frac{d^{2} x}{d t^{2}}+x \frac{2 f^{\prime \prime}\left(x^{2}\right)+x^{2} f^{\prime \prime \prime}\left(x^{2}\right)}{f^{\prime}\left(x^{2}\right)+x^{2} f^{\prime \prime}\left(x^{2}\right)}\left(\frac{d x}{d t}\right)^{2}=0 .
$$

This is equivalent to the following equation

$$
u t=\int_{0}^{x} \sqrt{f^{\prime}\left(s^{2}\right)+s^{2} f^{\prime \prime}\left(s^{2}\right)} d s,
$$

where $u$ is the norm of the initial velocity of $c ; u=\|\dot{c}(0)\|=\sqrt{f^{\prime}(0)}|\dot{x}(0)|$. If $f$ is given especially by

$$
f(s)=\int_{0}^{s} \frac{1}{s} \log (1+s) d s,
$$

then we have $x(t)=\sinh (u t)$. By spherical symmetry every geodesic para- 
metrized by arc-length emanating from $\tilde{o}$ is described by $\sinh t \cdot z, z \in T_{\tilde{o}} \mathrm{C}^{n}$ $\approx \mathbf{C}^{n},\|z\|=\sqrt{f^{\prime}(0)}|z|=|z|=1$. It follows that the exponential mapping $\exp _{\tilde{o}}$ at $\tilde{o}$ is a diffeomorphism of $T_{\tilde{o}} \mathbf{C}^{n}$ onto $\mathbf{C}^{n}$ and the minimizing geodesic from $\tilde{o}$ to $z$ is given by

$$
t \mapsto \sinh t \cdot z /|z|, \quad t \in[0, d(\tilde{o}, z)],
$$

hence $|z|=\sinh (d(\tilde{o}, z))$, that is, $\tilde{r}(z)=\sinh ^{-1}|z|$, where $\tilde{r}$ is the distance function measured from $\tilde{o}$.

Since $\tilde{g}$ is spherically symmetric, we may restrict the infimum of the sectional curvatures to $L$ for the computation of $F$. On the subspace $S=$ $\left.\operatorname{span}\left\{\partial / \partial z^{i}, \partial / \partial \bar{z}^{j} ; i, j>1\right\} \subset T \mathbf{C}^{n}\right|_{L}, \tilde{g}$ has constant holomorphic sectional curvature $-2 f^{\prime \prime}\left(|z|^{2}\right) / f^{\prime}\left(|z|^{2}\right)$. Then it follows that $K_{\tilde{\sigma}}$ lies between $-f^{\prime \prime}\left(|z|^{2}\right) / 2 f^{\prime}\left(|z|^{2}\right)$ and $-2 f^{\prime \prime}\left(|z|^{2}\right) / f^{\prime}\left(|z|^{2}\right)$ for any plane $\tilde{\sigma}$ in $S$ at $z \in L$. By spherical symmetry we may assume each plane not contained in $S$ to be spanned by real vectors of the form $X=a \cdot \partial / \partial z^{1}+\bar{a} \cdot \partial / \partial \bar{z}^{-1}, Y=b \cdot \partial / \partial z^{1}$ $+\bar{b} \cdot \partial / \partial \bar{z}^{1}+c \cdot \partial / \partial z^{2}+\bar{c} \cdot \partial / \partial \bar{z}^{2} ; a, b, c \in \mathbf{C}$. Then we have

$$
K_{X \wedge Y}=\frac{(a \bar{b}-b \bar{a})^{2} \alpha(z)-2|a|^{2}|b|^{2} \beta(z)}{-(a \bar{b}-b \bar{a})^{2} \gamma^{2}(z)+4|a|^{2}|c|^{2} f^{\prime}\left(|z|^{2}\right) \cdot \gamma(z)}
$$

where

$$
\begin{aligned}
\alpha(z)= & 2 f^{\prime \prime}\left(x^{2}\right)+4 x^{2} f^{\prime \prime \prime}\left(x^{2}\right)+x^{4} f^{(4)}\left(x^{2}\right) \\
& -x^{2} \frac{\left(2 f^{\prime \prime}\left(x^{2}\right)+x^{2} f^{\prime \prime \prime}\left(x^{2}\right)\right)^{2}}{f^{\prime}\left(x^{2}\right)+x^{2} f^{\prime \prime}\left(x^{2}\right)}, \\
\beta(z)= & f^{\prime \prime}\left(x^{2}\right)+x^{2} f^{\prime \prime \prime}\left(x^{2}\right)-x^{2} \frac{\left(f^{\prime \prime \prime}\left(x^{2}\right)\right)^{2}}{f^{\prime}\left(x^{2}\right)}
\end{aligned}
$$

and

$$
\gamma(z)=f^{\prime}\left(x^{2}\right)+x^{2} f^{\prime \prime}\left(x^{2}\right), \quad x=|z| \quad[2] .
$$

We may assume $a$ to be real by spherical symmetry. The curvature $K_{X \wedge Y}$ does not depend on the length of $Y$. Set $\|Y\|^{2}=2|b|^{2} \cdot \gamma(z)+2|c|^{2} f^{\prime}\left(z^{2}\right)=$ $2 \gamma(z)$ and $s=|b|^{2}, t=\sin ^{2}(\arg b)$. Then we have

$$
K_{X \wedge Y}=\frac{-4 \alpha(z) s t-2\left\{\beta(z) \cdot \gamma(z) / f^{\prime}\left(|z|^{2}\right)\right\}(1-s)}{4 \gamma^{2}(z)(1-s+s t)} .
$$

A troublesome computation yields that the infimum of $K_{X \wedge Y}$ over the set $\{(s, t) ; 0<s, t \leqslant 1,(s, t) \neq(0,1)\}$ is equal to $\operatorname{Min}\left\{-\beta(z) / 2 f^{\prime}\left(|z|^{2}\right) \gamma(z)\right.$, $\left.-\alpha(z) / \gamma^{2}(z)\right\}$. It follows that 
$\operatorname{Min}\left\{K_{\tilde{\sigma}} ; \tilde{\boldsymbol{\sigma}}\right.$ at $\left.z\right\}$

$$
=\operatorname{Min}\left\{-\frac{f^{\prime \prime}\left(|z|^{2}\right)}{2 f^{\prime}\left(|z|^{2}\right)},-\frac{2 f^{\prime \prime}\left(|z|^{2}\right)}{f^{\prime}\left(|z|^{2}\right)},-\frac{\beta(z)}{2 f^{\prime}\left(|z|^{2}\right) \gamma(z)},-\frac{\alpha(z)}{\gamma^{2}(z)}\right\} .
$$

If especially $f(s)=\int_{0}^{s} s^{-1} \log (1+s) d s$, it is written as

$$
\operatorname{Min}\left\{\frac{1}{1+|z|^{2}}, \frac{|z|^{2}-\log \left(1+|z|^{2}\right)}{2\left(1+|z|^{2}\right)\left\{\log \left(1+|z|^{2}\right)\right\}^{2}}\right\} \text {. }
$$

Since $|z|=\sinh (\tilde{r}(z))$, we have

$$
F(t)=\operatorname{Min}\left\{\frac{1}{\cosh ^{2} t}, \frac{\sinh ^{2} t-\log \left(\cosh ^{2} t\right)}{2 \cosh ^{2} t\left\{\log \left(\cosh ^{2} t\right)\right\}^{2}}\right\}
$$

Note that for some $t_{0}$,

$$
F(t)=\frac{\sinh ^{2} t-\log \left(\cosh ^{2} t\right)}{2 \cosh ^{2} t\left\{\log \left(\cosh ^{2} t\right)\right\}^{2}}
$$

for $t \leqslant t_{0}$ and $=1 /\left(\cosh ^{2} t\right), t \geqslant t_{0}$.

3. We will show the Theorem as follows. Since the exponential mapping $\exp _{\tilde{o}}: T_{\tilde{o}} \mathbf{C}^{n} \rightarrow \mathbf{C}^{n}$ is a diffeomorphism, there is no point on any geodesic emanating from $\tilde{o}$ which is conjugate to $\tilde{o}$. The sectional curvatures of $(M, g)$ and $\left(C^{n}, \tilde{g}\right)$ satisfy the condition (**). It follows by Rauch comparison theorem that there is also no point on any geodesic emanating from $o$ in $M$ which is conjugate to $o$, hence $\exp _{o}: T_{o} M \rightarrow M$ is a covering mapping [3]. From the simple connectivity of $M$, $\exp _{o}$ is a diffeomorphism. The distance function $r$ measured from $o$ is continuous on $M$ and smooth on $M \backslash\{o\}$. Note that $r^{2}$ is a smooth function.

We will show the existence of an appropriate smooth convex function $h$ of $r$ such that $h \circ r$ is proper and strictly plurisubharmonic over $M$ in the case where $(M, g)$ is Kählerian.

We remark that the Levi form $L(\varphi)$ of a smooth function $\varphi$ on $M$ is written as

$$
L(\phi)(v, \bar{v})=H(\phi)(X, X)+H(\varphi)(J X, J X), \quad v \in T_{p} M^{(1,0)},
$$

where $H(\varphi)$ is the Hessian of $\varphi, X=(v+v) / 2$.

It follows from (**) that

$$
H(r)(X, X) \geqslant H(\tilde{r})(\tilde{X}, \tilde{X}),
$$

where $X \in T_{c(t)} M$ and $\tilde{X} \in T_{\tilde{c}(t)} \mathbf{C}^{n},\|X\|=\|\tilde{X}\|=1, c$ and $\tilde{c}$ are rays starting at $o$ and $\tilde{o}$ respectively. The comparison theorem for the Hessian of the distance functions in [4] is stated under the assumption that the given 
manifolds are with nonpositive curvature. The nonpositivity of the curvatures is only used in order for the exponential mappings to be diffeomorphisms. Since the exponential mappings at $o$ and $\tilde{o}$ are diffeomorphisms in our case, the comparison theorem may be applied.

Set $h: s \rightarrow \sinh ^{2} s$. Then $h^{\prime}>0$ and $h^{\prime \prime}>0$ for $s>0$ and $h(\tilde{r}(z))=|z|^{2}$. The Hessian of $h \circ r$ is written as

$$
H(h \circ r)(X, X)=h^{\prime \prime}(t) H(r)(X, X)+h^{\prime}(t)|g(X, \partial / \partial r)|^{2}
$$

at $c(t)$. If we choose a unit vector $\tilde{X}$ at $\tilde{c}(t)$ in $\mathbf{C}^{n}$ such that $\tilde{g}(\tilde{X}, \partial / \partial \tilde{r})=$ $g(X, \partial / \partial r)$, then

$$
\begin{aligned}
H(h \circ r)(X, X) & >h^{\prime \prime}(t) H(\tilde{r})(\tilde{X}, \tilde{X})+h^{\prime}(t)|\tilde{g}(\tilde{X}, \partial / \partial \tilde{r})|^{2} \\
& =H(h \circ \tilde{r})(\tilde{X}, \tilde{X})>1\|\tilde{X}\|^{2}=1,
\end{aligned}
$$

hence by $(* * *) L(h \circ r)(v, \vec{v})=H(h \circ r)(X, X)+H(h \circ r)(J X, J X)>2$, that is, the Levi form of $h \circ r$ is positive definite. It follows that $h \circ r$ is strictly plurisubharmonic on $M \backslash\{o\}$. Since $h(r) \sim r^{2}+r^{4} / 3+\ldots$ at $r=$ $0, h \circ r$ is also strictly plurisubharmonic at $o$. By the definition of $h, h \circ r$ is proper. It is concluded from Grauert's theorem that $M$ is a Stein manifold.

\section{REFERENCES}

1. E. Cartan, Lecon sur la géométrie des expaces de Riemann, Gauthier-Villars, Paris, 1963.

2. P. F. Klembeck, A complete Kähler metric of pasitive curvature on $\mathbf{C}^{n}$, Proc. Amer. Math. Soc. 64 (1977), 313-316.

3. S. Kobayashi, Riemannian manifolds without conjugate points, Ann. Mat. Pura Appl. 53 (1961), 149-155.

4. Y. T. Siu and S. T. Yau, Complete Kähler manifolds with nompasitive curvature of faster than quadratic decay, Ann. of Math. 105 (1977), 225-264.

5. H. H. Wu, Negatively curved Kähler manifolds, Notices Amer. Math. Soc. 14 (1967), 515.

INSTITUTE OF MATHEMATICS, UNIVERSITY OF TSUKUBA, IBARAKI 300-31, JAPAN 